\title{
OSCILLATION AND NONOSCILLATION IN A NONAUTONOMOUS DELAY-LOGISTIC EQUATION*
}

BY

B. G. ZHANG' (Shandong College of Oceanography, People's Republic of China)

AND

K. GOPALSAMY ${ }^{2}$ (The Flinders University of South Australia)

Abstract. Sufficient conditions are obtained for the delay-logistic equation $\dot{x}(t)=$ $r(t) x(t)[1-x(t-\tau(t)) / K]$ to be respectively oscillatory and nonoscillatory.

1. Introduction. The purpose of this article is to derive sufficient conditions for the nonautonomous delay-logistic equation

$$
\frac{d x(t)}{d t}=r(t) x(t)\left[1-\frac{x(t-\tau(t))}{K}\right], \quad t>0
$$

to be respectively "oscillatory" and "nonoscillatory" where $r$ and $\tau$ are positive continuous functions defined on $[0, \infty)$ and $K$ is a positive constant. When $r, \tau, K$ are positive constants, (1.1) is known as Hutchinson's equation in the literature on mathematical ecology and has been discussed extensively by several authors and notably by Wright [9], Jones [3], Kakutani and Markus [4]. The oscillatory nature of an autonomous equation of the form

$$
\frac{d u(t)}{d t}=u(t)\left[a-\sum_{j=1}^{n} b_{j} u\left(t-\tau_{j}\right)\right]
$$

where $a, b_{j}, \tau_{j}(j=1,2, \ldots, n)$ are positive constants has been recently discussed by Gopalsamy [2] and Kulenovic et al. [6]. The literature on oscillation of scalar systems with deviating arguments is quite extensive and we refer to the books by Shevelo [8] and Lakshmikantham et al. [7] for more details.

Motivated by the plausible applications of (1.1), we consider solutions of (1.1) corresponding to initial conditions of the type

$$
\begin{aligned}
x(s)=\varphi(s) \geq 0, \quad \varphi(0) & >0, \quad \varphi \text { is continuous on }\left[-\tau^{*}, 0\right], \\
\tau^{*} & =\sup _{t \geq 0} \tau(t) \leq \infty .
\end{aligned}
$$

* Received December 20, 1986.

'Department of Mathematics, Shandong College of Oceanography, Qingdao, Shandong, The People's Republic of China.

${ }^{2}$ School of Mathematical Sciences, The Flinders University of South Australia, Bedford Park, S. A. 5042, Australia. 
Since $\tau(t)>0$ for $t>0$, it is not difficult to show as in $\mathrm{El}^{\prime} \mathrm{sgol}^{\prime} \mathrm{c}$ and Norkin [1, pp. 10-11] that solutions of (1.1) and (1.3) are defined for all $t \geq 0$ and also remain positive for $t \geq 0$. A solution $x_{\varphi}(\cdot)$ of $(1.1)$ and (1.3) is said to be "oscillatory about $K$ " if and only if there exists a sequence $\left\{t_{n}\right\} \rightarrow \infty$ as $n \rightarrow \infty$ such that $x\left(t_{n}\right)-K=0$ for each $t_{n} \in\left\{t_{n}\right\}$. We introduce a change of variables in (1.1) so that

$$
y(t)=\frac{x(t)}{K}-1, \quad t \geq-\tau^{*}
$$

and note that $y$ is governed by

$$
\frac{d y(t)}{d t}=-r(t)[1+y(t)] y(t-\tau(t)), \quad t>0,
$$

whose initial conditions are inherited from (1.3) through (1.4). The oscillation of $x$ about $K$ is now equivalent to that of $y$ about zero as in the usual case. We note from the positivity of $x(t)$ for $t \geq 0$ that $1+y(t)>0$ for $t \geq 0$.

2. Oscillation and nonoscillation. It is trivial to verify that if $\tau(t) \equiv 0$, then every solution of (1.5) and hence of (1.1) is monotonic in $t$ and hence any oscillation in (1.5) is induced by the delay in the argument. Our first result below is concerned with the asymptotic behaviour of solutions of (1.5).

THEOREM 2.1. Assume the following:

(i) $r, \tau$ are continuous positive functions defined on $[0, \infty)$;

(ii) $t-\tau(t) \rightarrow \infty$ as $t \rightarrow \infty$;

(iii) for some $t_{0} \geq 0, \int_{t_{0}}^{\infty} r(s) d s=\infty$.

Then every solution of (1.5) is either oscillatory or converges to zero monotonically for large $t$ as $t \rightarrow \infty$.

Proof. Suppose that $y(t)>0$ for $t \geq T$. It follows from (1.5) and (2.1), $\dot{y}(t)<0$ for $t>T^{*}$ where $T^{*}>T$ such that $T^{*}-\tau\left(T^{*}\right)>T$ and hence

$$
\lim _{t \rightarrow \infty} y(t)=\alpha \geq 0 \text { exists. }
$$

If $\alpha>0$ then we have from (1.5),

$$
\dot{y}(t) \leq-\alpha(1+\alpha) r(t) \quad \text { for } t \geq T^{*}
$$

leading to

$$
y(\infty)-y\left(t^{*}\right) \leq-\alpha(1+\alpha) \int_{t^{*}}^{\infty} r(s) d s
$$

where $t^{*}=\max \left\{t_{0}, T^{*}\right\}$. But (2.5) contradicts (2.2). Suppose now that $y(t)<0$ for $t \geq T$. Since (1.5) implies $\dot{y}(t)>0$ for $t \geq T^{*}$,

$$
\lim _{t \rightarrow \infty} y(t)=\beta \leq 0 \text { exists. }
$$

If $\beta<0$, we have

$$
\begin{aligned}
\dot{y}(t) & \geq-[1+y(0)] y(t-\tau(t)) r(t) \\
& \geq-[1+y(0)] \beta r(t)
\end{aligned}
$$


which again on integration over $\left[t^{*}, \infty\right)$ leads to a contradiction of $(2.2)$. This completes the proof.

Our next result provides a sufficient condition for all nontrivial solutions of (1.5) to be oscillatory.

THEOREM 2.2. In addition to assumptions of Theorem 2.1 assume

$$
\liminf _{t \rightarrow \infty} \int_{t-\tau(t)}^{t} r(s) d s>1 / e .
$$

Then every nontrivial solution of (1.5) is oscillatory.

Proof. We define $\delta(t)$ as follows:

$$
\delta(t)=\max _{s \in[0, t]}\{s-\tau(s)\}
$$

and note that $(2.8)$ implies

$$
\liminf _{t \rightarrow \infty} \int_{\delta(t)}^{t} r(s) d s>1 / e
$$

In fact if (2.10) does not hold, then there exists a sequence $\left\{t_{n}\right\} \rightarrow \infty$ as $n \rightarrow \infty$ such that

$$
\lim _{n \rightarrow \infty} \int_{\delta\left(t_{n}\right)}^{t_{n}} r(s) d s \leq 1 / e .
$$

But from (2.9) we have

$$
\delta\left(t_{n}\right)=\max _{\delta \in\left[0, t_{n}\right]}\{s-\tau(s)\}
$$

and hence there exists $t_{n}^{\prime} \in\left[0, t_{n}\right]$ such that

$$
\delta\left(t_{n}\right)=t_{n}^{\prime}-\tau\left(t_{n}^{\prime}\right)=\max _{\delta \in\left[0, t_{n}\right]}\{s-\tau(s)\} .
$$

Hence we have

$$
\int_{t_{n}^{\prime}-\tau\left(t_{n}^{\prime}\right)}^{t_{n}^{\prime}} r(s) d s \leq \int_{\delta\left(t_{n}\right)}^{t_{n}} r(s) d s, \quad n=1,2,3, \ldots
$$

showing that

$$
\left\{\int_{t_{n}^{\prime}-\tau\left(t_{n}^{\prime}\right)}^{t_{n}^{\prime}} r(s) d s\right\} \quad(n=1,2,3, \ldots)
$$

is a bounded sequence having a convergent subsequence, say

$$
\left\{\int_{t_{n_{k}}^{\prime}-\tau\left(t_{n_{k}}^{\prime}\right)}^{t_{n_{k}}^{\prime}} r(s) d s\right\} \rightarrow c \leq 1 / e \quad \text { as } k \rightarrow \infty .
$$

But this implies that

$$
\liminf _{t \rightarrow \infty} \int_{t-\tau(t)}^{t} r(s) d s \leq 1 / e
$$

which contradicts (2.8). Suppose now the assertion of Theorem 2.2 is false. Then there exists a nonoscillatory solution, say $y$, of (1.4) such that

$$
y(t)>0, \quad y(t-\tau(t))>0 \quad \text { for } t>T^{*} .
$$


A consequence of $(2.11)$ is that the linear delay differential inequality

$$
\dot{y}(t)+r(t) y(t-\tau(t)) \leq 0
$$

has an eventually positive solution when (2.8) holds. But it is known that this is not possible by a result of Koplatadze and Chanturiya [5].

Let us now suppose that $(1.5)$ has an eventually negative solution $y(t)<0$ for $t \geq T$. Since $1+y(t)>0, \dot{y}(t)>0$ for $t>T^{*}$, we have

$$
\begin{aligned}
\dot{y}(t) & =-r(t)[1+y(t)] y(t-\tau(t)) \\
& \geq-r(t)[1+y(t)] y(\delta(t))
\end{aligned}
$$

and hence

$$
\int_{\delta(t)}^{t} \frac{\dot{y}}{y} d t \leq-\int_{\delta(t)}^{t} r(s)[1+y(s)] \frac{y(\delta(s))}{y(s)} d s
$$

implying

$$
\ln \frac{y(\delta(t))}{y(t)} \geq \int_{\delta(t)}^{t} r(s)[1+y(s)] \frac{y(\delta(s))}{y(s)} d s .
$$

Let $w$ be defined by

$$
w(t)=\frac{y(\delta(t))}{y(t)}, \quad t>T^{*}
$$

and note that $w(t) \geq 1$ since $\dot{y}(t)>0$ for $t \geq T^{*}$. From (2.15) and (2.16),

$$
\ln w(t) \geq w(\xi) \int_{\delta(t)}^{t} r(s)[1+y(s)] d s \quad \text { for } \xi \in(\delta(t), t) .
$$

We shall show that $w$ is bounded; by Theorem $2.1, y(t) \rightarrow 0$ as $t \rightarrow \infty$ since $y$ is nonoscillatory. Hence for large enough $T^{*}$,

$$
1+y(t) \geq \frac{1}{2}, \quad \int_{\delta(t)}^{t} r(s) d s \geq c>\frac{1}{e} \quad \text { for } t>T^{*} .
$$

For any $t^{*} \geq T^{*}$, there exists a $t \in\left[\delta\left(t^{*}\right), t^{*}\right)$ such that

$$
\int_{\delta\left(t^{*}\right)}^{t} r(s) d s \geq \frac{c}{2}, \quad \int_{t}^{t^{*}} r(s) d s \geq \frac{c}{2} .
$$

We have from (1.5),

$$
\begin{aligned}
y(t)-y\left(\delta\left(t^{*}\right)\right) & \geq-\int_{\delta\left(t^{*}\right)}^{t} r(s)[1+y(s)] y(\delta(s)) d s \\
& \geq \frac{1}{2}[-y(\delta(t))] \int_{\delta\left(t^{*}\right)}^{t} r(s) d s \\
& \geq \frac{c}{4}[-y(\delta(t))]
\end{aligned}
$$

and hence

$$
y\left(\delta\left(t^{*}\right)\right) \leq \frac{c}{4} y(\delta(t)) .
$$

Similarly again from (1.5),

$$
y\left(t^{*}\right)-y(t) \geq \frac{c}{4}\left[-y\left(\delta\left(t^{*}\right)\right)\right]
$$


implying

$$
y(t) \leq \frac{c}{4} y\left(\delta\left(t^{*}\right)\right) \leq\left(\frac{c}{4}\right)^{2} y(\delta(t)) .
$$

Since $y(t)<0$, we have from $(2.22)$,

$$
w(t)=\frac{y(\delta(t))}{y(t)} \leq\left(\frac{4}{c}\right)^{2}
$$

from which the boundedness of $w$ follows. We define

$$
l=\liminf _{t \rightarrow \infty} w(t), \quad l<\infty .
$$

Taking $\lim \inf _{t \rightarrow \infty}$ on both sides of $(2.15)$,

$$
\frac{\ln l}{l} \geq \liminf _{t \rightarrow \infty} \int_{\delta(t)}^{t} r(s) d s
$$

leading to

$$
\liminf _{t \rightarrow \infty} \int_{\delta(t)}^{t} r(s) d s \leq 1 / e
$$

which contradicts (2.10) and hence (2.8). This completes the proof.

A sufficient condition for the existence of a nonoscillatory solution of (1.5) is formulated as follows:

THEOREM 2.3. Let $r, \tau$ be continuous positive functions on $[0, \infty)$ such that

$$
\limsup _{t \rightarrow \infty} \int_{t-\tau(t)}^{t} r(s) d s<1 / e .
$$

Then (1.5) has a nonoscillatory solution on $[0, \infty)$.

Proof. Our proof is based on an application of the well-known Schauder-Tychonoff fixed point theorem. Let $C\left[t_{0}, \infty\right)$ denote a locally convex linear space of all continuous real-valued functions on $\left[t_{0}, \infty\right)$ endowed with the topology of uniform convergence on compact subsets of $\left[t_{0}, \infty\right)$. Define a set $S$ as follows:

$$
S=\left\{\begin{array}{l|l}
y \in C\left[t_{0}, \infty\right) & \begin{array}{l}
y \text { is nondecreasing on }\left[t_{0}, \infty\right), \\
-(1-\varepsilon) \leq y(t) \leq-(1-\varepsilon) \exp \left[-e \int_{t_{1}}^{t} r(s) d s\right], \quad t \geq t_{1}, \\
y(t) \equiv-(1-\varepsilon) \text { on }\left[t_{0}, t_{1}\right], \\
y(t) e \leq y(t-\tau(t)) \text { for } t \geq t_{1},
\end{array}
\end{array}\right\}
$$

where $t_{1}$ is sufficiently large such that $\int_{t-\tau(t)}^{t} r(s) d s \leq 1 / e$, for $t \geq t_{1}$ and $\varepsilon$ is a fixed positive number such that $1-\varepsilon>0$. We note that $S$ is a nonempty closed convex subset of $C\left[t_{0}, \infty\right)$. We define a map $F: S \rightarrow C\left[t_{0}, \infty\right)$ as follows:

$$
F(y)(t)=\left\{\begin{array}{l}
-(1-\varepsilon), \quad t \in\left[t_{0}, t_{1}\right] \\
-(1-\varepsilon) \exp \left[-\int_{t_{1}}^{t} \frac{r(s)[1+y(s)] y(s-\tau(s))}{y(s)} d s\right], \quad t \geq t_{1} .
\end{array}\right.
$$

We first verify $F S \subset S$; it is easy to see that

$$
F(y)(t) \geq-(1-\varepsilon) \text { for } t \geq t_{0}
$$


and

$$
\begin{aligned}
& \int_{t_{1}}^{t} \frac{r(s)[1+y(s)] y(s-\tau(s))}{y(s)} d s \\
& \quad \leq e \int_{t_{1}}^{t} r(s)\left[1-(1-\varepsilon) \exp \left(-e \int_{t_{1}}^{s} r(u) d u\right)\right] d s \\
& \quad \leq e \int_{t_{1}}^{t} r(s) d s .
\end{aligned}
$$

From (2.30) and (2.31),

$$
-(1-\varepsilon) \leq F(y)(t) \leq-(1-\varepsilon) \exp \left[-e \int_{t_{1}}^{t} r(s) d s\right] \text { for } t \geq t_{1}
$$

It is also found that

$$
\begin{aligned}
\frac{F(y)(t)}{F(y)(t-\tau(t))} & =\exp \left[-\int_{t-\tau(t)}^{t} \frac{r(s)[1+y(s)] y(s-\tau(s))}{y(s)} d s\right] \\
& \geq(1 / e) \quad \text { for } t \geq t_{1} .
\end{aligned}
$$

It follows from the above, $F S \subset S$. The continuity of $F: S \rightarrow S \subset C\left[t_{0}, \infty\right)$ is verified as follows: let $y_{n} \in S, y \in S$ and let $y_{n} \rightarrow y$ as $n \rightarrow \infty$. Let $t_{2}$ be a fixed number such that $t_{1}<t_{2}<\infty$. We have from the uniform convergence of $y_{n} \rightarrow y$ on $\left[t_{1}, t_{2}\right]$ that for any $\varepsilon_{1}>0$ there exists $n_{0}\left(\varepsilon_{1}\right)$ satisfying

$$
\sup _{s \in\left[t_{1}, t_{2}\right]}\left|\frac{\left[1+y_{n}(s)\right] y_{n}(s-\tau(s))}{y_{n}(s)}-\frac{[1+y(s)] y(s-\tau(s))}{y(s)}\right|<\varepsilon_{1} \quad \text { for } n>n_{0}\left(\varepsilon_{1}\right) .
$$

From the definition of $F$, for $t \in\left[t_{1}, t_{2}\right]$,

$$
\begin{aligned}
\mid F\left(y_{n}\right)(t)- & F(y)(t)|=(1-\varepsilon)| \exp \int_{t_{1}}^{t} \frac{r(s)\left[1+y_{n}(s)\right] y_{n}(s-\tau(s))}{y_{n}(s)} d s \\
& \quad-\exp \int_{t_{1}}^{t} \frac{r(s)[1+y(s)] y(s-\tau(s))}{y(s)} d s \mid \\
\leq & (1-\varepsilon) \int_{t_{1}}^{t} r(s)\left|\frac{\left[1+y_{n}(s)\right] y_{n}(s-\tau(s))}{y_{n}(s)}-\frac{[1+y(s)] y(s-\tau(s))}{y(s)}\right| d s \\
& \leq(1-\varepsilon) \varepsilon_{1} \int_{t_{1}}^{t} r(s) d s \text { for } n>n_{0}\left(\varepsilon_{1}\right) \\
& \leq(1-\varepsilon) \varepsilon_{1} \int_{t_{1}}^{t_{2}} r(s) d s .
\end{aligned}
$$

Since $\varepsilon_{1}$ is arbitrary, the continuity of $F$ on $S$ follows. It is easy to see that $\left|\frac{d}{d t} F(y)(t)\right|$ is uniformly (in $y$ ) bounded for $t$ on $\left[t_{1}, \infty\right.$ ) showing the equiboundedness of the family $F S$. Now by the Arzela-Ascoli theorem, the precompactness of $F S$ follows. All the requirements of the Schauder-Tychonoff fixed point theorem are satisfied and hence there exists a $y^{*} \in S$ such that $F\left(y^{*}\right)(t)=y^{*}(t)$. It is easy to see that this $y^{*}$ is a nonoscillatory solution of $(1.5)$ if we identify $t_{0}$ of $C\left[t_{0}, \infty\right)$ with $-\tau^{*}$. The proof is complete. 
3. Some remarks. We note that our sufficient conditions for the oscillation and nonoscillation of (1.5) are sharp in the following sense: if $r$ and $\tau$ are positive constants respectively equal to $r_{0}$ and $\tau_{0}$, it is known that

$$
r_{0} \tau_{0}>1 / e
$$

is a necessary and sufficient condition for all solutions of

$$
x(t)=r_{0} x(t)\left[1-\frac{x\left(t-\tau_{0}\right)}{K}\right]
$$

to be oscillatory about $K$ (see Kakutani and Markus [4]). Our sufficiency condition of Theorem 2.3 for the nonoscillation of (1.5) and hence of (1.1) leads to

$$
r_{0} \tau_{0} \leq 1 / e
$$

in the autonomous case and it is known that (3.3) is a necessary condition for the nonoscillation of (3.2). Thus the sufficient condition of Theorem 2.3 is also sharp. It is not known whether the conditions (2.8) and (2.27) are necessary in the nonautonomous case for the respective results.

\section{REFERENCES}

[1] L. Ė. Èl'sgol'c and S. B. Norkin, Introduction to the theory and application of differential equations with deviating arguments, Mathematics in Science and Engineering, vol. 105, Academic Press, New York, 1973

[2] K. Gopalsamy, Oscillations in a delay-logistic equation, Quart. Appl. Math. 44, 447-461 (1986)

[3] G. S. Jones, The existence of periodic solutions of $f^{\prime}(x)=-\alpha f(x-1)[1+f(x)]$, J. Math. Anal. Appl. 5, 435-450 (1962)

[4] S. Kakutani and L. Markus, On the nonlinear difference-differential equation $y^{\prime}(t)=[A-B y(t-$ $\tau)] y(t)$ in Contributions to the theory of nonlinear oscillations, IV, Annals of Mathematics Study 41, Princeton University Press, N. J., 1958

[5] R. G. Koplatadze and T. A. Chanturiya, Oscillating and monotone solutions of first-order differential equations with deviating argument, Differential Equations 18, 1463-1465 (1982)

[6] M. R. S. Kulenović, G. Ladas, and A. Meimaridou, On oscillation of nonlinear delay differential equations, Quart. Appl. Math. 45, 155-164 (1987)

[7] V. Lakshmikantham, G. S. Ladde, and B. Zhang, Oscillation theory of differential equations with deviating arguments, Marcel Dekker Inc. (to appear)

[8] V. N. Shevelo, Oscillation of solutions of differential equations with deviating arguments, Naukova Dumka, Kiev, 1972

[9] E. M. Wright, A nonlinear difference-differential equation, J. Reine Angew. Math. 194, 66-87 (1955) 